\title{
Phenolic acids, cinnamic acid, and ergosterol as cosmeceutical ingredients: Stabilization by microencapsulation to ensure sustained bioactivity
}

\author{
Oludemi Taofiq ${ }^{\mathrm{a}, \mathrm{b}, \mathrm{c}}$, Sandrina A. Heleno ${ }^{\mathrm{a}, \mathrm{b}}$, Ricardo C. Calhelha ${ }^{\mathrm{a}}$, Isabel P. Fernandes ${ }^{\mathrm{a}, \mathrm{b}}$, \\ Maria José Alves ${ }^{\mathrm{a}}$, Lillian Barros ${ }^{\mathrm{a}, \mathrm{b}}$, Ana M. González-Paramás ${ }^{\mathrm{c}}$, Isabel C.F.R. Ferreira ${ }^{\mathrm{a}, *}$, \\ Maria F. Barreiro ${ }^{\mathrm{a}, \mathrm{b}, * *}$ \\ ${ }^{a}$ Centro de Investigação de Montanha (CIMO), Instituto Politécnico de Bragança, Campus de Santa Apolónia, 5300-253 Bragança, Portugal \\ ${ }^{\mathrm{b}}$ Laboratory of Separation and Reaction Engineering (LSRE), Associate Laboratory LSRE/LCM, Polytechnic Institute of Bragança, Campus de Santa Apolónia, 1134, 5301- \\ 857 Bragança, Portugal \\ ${ }^{\mathrm{c}}$ GIP-USAL, Unidad de Nutrición y Bromatología, Faculty of Pharmacy, University of Salamanca, Campus Miguel de Unamuno, 37007 Salamanca, Spain
}

\section{A R T I C L E I N F O}

\section{Keywords:}

Bioactive compounds

Cosmeceuticals

Bioactive properties

Microencapsulation

Controlled release

\begin{abstract}
A B S T R A C T
The global cosmetic industry is constantly searching for new ingredients with multifunctional properties. In this context, phenolic compounds ( $p$-hydroxybenzoic, $p$-coumaric, and protocatechuic acids), cinnamic acid and ergosterol were evaluated for their anti-inflammatory, anti-tyrosinase and antimicrobial activities, and thereafter microencapsulated by the atomization/coagulation technique. After characterization (morphology, particle size distribution and encapsulation efficiency), the microencapsulated compounds were incorporated into a semi-solid base cream and their performance evaluated comparatively with the use of the free forms. The cosmeceutical formulations were checked regarding the presence of the bioactive compounds by HPLC-DAD, and for their physicochemical properties (colour and $\mathrm{pH}$ ). The obtained results demonstrated the anti-tyrosinase, anti-inflammatory and antimicrobial activities of the individual compounds that, after incorporation, have shown a decreasing pattern along time. On the contrary, the formulations incorporating the microencapsulated bioactive compounds gave rise to a gradual release, ensuring bioactivity maintenance. These results underline the advantage of using microencapsulation to preserve and ensure the controlled release of bioactive species in cosmeceutical formulations.
\end{abstract}

\section{Introduction}

Skin care formulations are designed to exert multifunctional properties, thus increasing the industrial interest of using bioactive agents such as phenolic compounds, vitamins, terpenoids, steroids and polyunsaturated fatty acids from natural sources [1]. The use of bioactive compounds as cosmetic ingredients has generated a new product's trend known as "Cosmeceuticals", which is still unfamiliar to most of the global consumers. Cosmeceuticals are formulated with biologically active ingredients, that exert a medical drug-like benefit on the skin [2]. The skin is equipped with intrinsic enzymatic antioxidants which neutralize free radicals and repair oxidative damage. Continuous oxidative processes in the body lowers the activities of these antioxidants by promoting the skin ageing process [3]. Among the different classes of bioactive compounds used in skin care products, compounds that display antioxidant activity seem to be the most required due to their action on several cell signal transduction pathways related to ageing, inflammation, hyperpigmentation and extracellular matrix degradation [3-5].

Phenolic acids and related compounds, are a group of secondary metabolites from fungi and plants [6]. These compounds are known for their antioxidant, anti-collagenase, anti-inflammatory, antimicrobial and anti-tyrosinase activities, as well as for the ultraviolet (UV) protective effects making them appropriate multifunctional ingredients to be used in the design of topical formulations $[1,7]$. On the other hand, ergosterol is a precursor of vitamin D commonly found in fungi and protozoans' membranes. Numerous reports have suggested that ergosterol, ergosterol peroxide, ergostatrien-3 $\beta$-ol, hericirine, botulin, trametenolic acid and other steroidal compounds display biological properties such as antitumor [8], antioxidant [9] anti-inflammatory

\footnotetext{
* Corresponding author.

** Correspondence to: M.F. Barreiro, Laboratory of Separation and Reaction Engineering (LSRE), Associate Laboratory LSRE/LCM, Polytechnic Institute of Bragança, Campus de Santa Apolónia, 1134, 5301-857 Bragança, Portugal.

E-mail addresses: iferreira@ipb.pt (I.C.F.R. Ferreira), barreiro@ipb.pt (M.F. Barreiro).
} 
[10] and anti-tyrosinase activities [11]. Nevertheless, their use as bioactive ingredients in cosmeceutical formulations has not been yet fully established. Besides being already used in cosmetics due to their effectiveness in suppressing the severity of inflammatory diseases, psoriasis and eczema [7,12], sterols still need to be further studied to fully explore their cosmeceutical potential.

The use of these compounds in cosmeceutical formulations still face constraints related with instability, sensitivity to $\mathrm{pH}$ and temperature, easy degradation and low efficacy. To overcome these issues, recent trends pointed out the use of polymeric carriers such as chitosan, alginate and polylactic acids to protect (encapsulate) bioactive compounds [3,13]. Comparatively with ergosterol, and despite the fact that phenolic acids potential as cosmetic ingredients are well reported, very few studies were conducted to describe the incorporation of these molecules into cosmetics (e.g. semi solid base formulations), namely to evaluate their bioactive properties, stability, and maintenance over an extended period of time.

The present study intends to evaluate the anti-inflammatory, antimicrobial and anti-tyrosinase activities of $p$-hydroxybenzoic, $p$-coumaric, protocatechuic and cinnamic acids, and ergosterol. Based on the exhibited bioactivity, and interest to be used in cosmeceuticals formulations, these compounds were microencapsulated using the atomization/coagulation method with calcium alginate as the matrix material. The produced microspheres were fully characterised and tested against the use of the non-encapsulated forms (free compounds) in a base cosmetic (semi-solid formulation). Each produced formulation was submitted to bioactivity evaluation over a period of 6 months to ascertain stability and monitor the in vitro release of compounds responsible for the effect. Additionally, target compounds (phenolic acids, cinnamic acid, and ergosterol), incorporated in its free form were monitored using the HPLC-DAD technique.

\section{Materials and methods}

\subsection{Standards and reagents}

Acetonitrile 99.9\% was of high-performance liquid chromatography (HPLC) grade from Lab-Scan (Lisbon, Portugal), methanol was of analytical grade and supplied by Pronalab (Lisbon, Portugal). Dulbecco's modified Eagle's minimum essential medium (DMEM), $p$-hydroxybenzoic, $p$-coumaric, protocatechuic and cinnamic acids, fetal bovine serum (FBS), penicillin, streptomycin, Griess reagent system (Promega), DIMETHYL sulphoxide (DMSO), lipopolysaccharide (LPS), 3,4-dihydroxy-L-phenylalanine (L-DOPA) and mushroom tyrosinase enzyme were obtained from Sigma-Aldrich Co. (Saint Louis, MO, USA). Sodium alginate was provided by Fluka Chemie (USA), Calcium chloride and Tween 80 by Panreac (Spain). The culture media Muller Hinton broth (MHB) and Tryptic Soy Broth (TSB) were obtained from Biomerieux (Marcy l'Etoile, France). Blood agar with 7\% sheep blood and MacConkey agar plates were purchased from Biomerieux Marcy l'Etoile, France. The dye $p$-iodonitrotetrazolium chloride (INT) was purchased from Sigma-Aldrich (Spruce Street; St. Louis, MO, USA) and was used as a microbial growth indicator.

\subsection{Evaluation of bioactive properties of individual compounds}

\subsubsection{Anti-inflammatory activity}

The evaluation of the anti-inflammatory activity was carried out according to the procedure reported by Taofiq et al. [14]. Briefly, mouse macrophage-like cell line RAW264.7 was cultured in DMEM medium supplemented with $10 \%$ heat-inactivated fetal bovine serum, glutamine, and antibiotics at $37^{\circ} \mathrm{C}$ under $5 \% \mathrm{CO}_{2}$, in humidified air. For each experiment, cells were detached with a cell scraper. A cell density of $5 \times 10^{5}$ cells $/ \mathrm{mL}$ was used, and the proportion of dead cells was $<$ $5 \%$, according to Trypan blue dye exclusion test. Cells were seeded in 96-well plates at 150,000 cells/well and allowed do attach to the plate overnight. Then, cells were treated with different concentrations of each extract for $1 \mathrm{~h}$, and further stimulated with LPS $(1 \mu \mathrm{g} / \mathrm{mL})$ for $18 \mathrm{~h}$. Dexamethasone $(50 \mu \mathrm{M})$ was used as a positive control. The effect of all the tested samples in the absence of LPS was evaluated to observe possible induced changes in nitric oxide (NO) basal levels. Both compounds and LPS were dissolved in supplemented DMEM. For inhibition of NO, compounds were dissolved in 50\% DMSO and further submitted to sequential dilutions ( 400 to $50 \mu \mathrm{g} / \mathrm{mL}$ ) in order to determine the effective concentration necessary to reveal anti-inflammatory activity. Griess Reagent System kit which contains sulphanilamide, $N$-(1-napthyl) ethylenediamine hydrochloride (NED) and nitrite solutions was used. A reference curve of nitrite (sodium nitrite $100 \mu \mathrm{M}$ to $1.6 \mu \mathrm{M}$; $\left.y=0.0066 x+0.1349 ; R^{2}=0.9986\right)$ was prepared and the cell culture supernatant $(100 \mu \mathrm{L})$ was transferred to the plate and mixed with sulphanilamide and NED solutions, 5-10 min each, at room temperature. The NO production was determined by measuring the absorbance at $540 \mathrm{~nm}$ (microplate reader ELX800 Biotek), and by comparison with the standard calibration curve.

\subsubsection{Antimicrobial activity}

2.2.2.1. Selected microbial strains. The microbial strains used in the present work were clinical isolates obtained from patients hospitalized in various departments at the Hospital Center of Trás-os-Montes and Alto Douro (Vila Real, Portugal). Two Gram-negative bacteria (Escherichia coli and Proteus mirabilis (isolated from urine), and three Gram-positive bacteria (Enterococcus faecalis (isolated from urine), methicillin-sensitive Staphylococcus aureus (MSSA) (isolated from wound exudate), and methicillin-resistant Staphylococcus aureus (MRSA) (isolated from expectoration)), and a yeast, Candida albicans (isolated from urine) were tested. All the microorganisms were incubated in appropriate fresh medium for $48 \mathrm{~h}$ and kept in the oven at $37^{\circ} \mathrm{C}$ before analysis in order to maintain the exponential growth phase.

2.2.2.2. Characterization of the antibiotic susceptibility of the microbial strains. The microorganism identification and respective susceptibility to antibiotics was evaluated using MicroScan panels (MicroScan ${ }^{\circledR}$; Siemens Medical Solutions Diagnostics, West Sacramento, CA, USA) by the microdilution method. The results were analysed following the Interpretive Breakpoints as recommended by the Clinical and Laboratory Standards Institute (CLSI) [15]; Committee of L'Antibiogramme de la Société Française de Microbiologie (CA-SFM) [16]; and the European Committee on Antimicrobial Susceptibility Testing (EUCAST) [17] (Tables S1, S2, Supplementary materials).

2.2.2.3. Determination of the minimal inhibitory concentration (MIC). Each compound was dissolved in $25 \%$ DMSO to prepare a stock solution of at $25 \mathrm{mg} / \mathrm{mL}$ from which several dilutions were performed to establish a concentration test range between 0.078 and $5 \mathrm{mg} / \mathrm{mL}$. The determination of the minimal inhibitory concentration (MIC) was performed by the microdilution method and the rapid $p$ iodonitrotetrazolium chloride (INT) colourimetric assay, as also reported by Taofiq, Heleno et al. [18]. Initially, $100 \mu \mathrm{L}$ of the stock solution, $400 \mu \mathrm{L}$ of medium TSB or MHB, according to the bacteria requirements, were added making a solution of $5 \mathrm{mg} / \mathrm{mL}$ at $5 \%$ DMSO. Then, $190 \mu \mathrm{L}$ of this solution was added to each well of the 96 -well microplate, and therefore submitted to successive dilutions over the wells containing $90 \mu \mathrm{L}$ of MHB or TSB media. Then, $10 \mu \mathrm{L}$ of inoculum $\left(1.5 \times 10^{8} \mathrm{CFU} / \mathrm{mL}\right)$ were added to all the microplate wells. Two negative controls were prepared (one with MHB/TSB 5\% DMSO, and another one with the tested compounds and media). A positive control with MHB/TSB medium at 5\% DMSO and the inoculum was used as positive control. Regarding the antibiotic controls, colistin was used for the Gram-negative bacteria, vancomycin for the Gram-positive bacteria, and fluconazole for $C$. albicans, at a concentration based on the obtained MIC levels (Table S2, Supplementary materials). The plates were then 
incubated at $37^{\circ} \mathrm{C}$, for $24 \mathrm{~h}$, in an oven (Jouan, Berlin, Germany) and the MIC values determined after adding INT $(0.2 \mathrm{mg} / \mathrm{mL}, 40 \mu \mathrm{L})$ and after incubation at $37^{\circ} \mathrm{C}$ for $30 \mathrm{~min}$. Viable microorganisms reduced the yellow dye to a pink colour. MIC was defined as the lowest compound concentration that prevented this change, thus showing the inhibition of the microbial growth.

\subsubsection{Anti-tyrosinase activity}

The tyrosinase inhibition assay was performed using L-DOPA as substrate according to the procedure described by Taofiq, Heleno et al. [18]. The assay was carried out in triplicate in a 96-well microplate and absorbance was measured at $475 \mathrm{~nm}$. Compounds and ascorbic acid used as control were dissolved in 50\% DMSO. Each well contained $40 \mu \mathrm{L}$ of individual compound with $80 \mu \mathrm{L}$ of phosphate buffer $(0.1 \mathrm{M}$, $\mathrm{pH} 6.8), 40 \mu \mathrm{L}$ of tyrosinase enzyme ( $60 \mathrm{units} / \mathrm{mL}$ ), and $40 \mu \mathrm{L}$ of $\mathrm{L}$ DOPA ( $3.5 \mathrm{mM})$. The mixture was further incubated for $10 \mathrm{~min}$ at $37^{\circ} \mathrm{C}$, and the absorbance (A) was measured at $475 \mathrm{~nm}$ using a microplate reader (ELX800 Biotek). Each sample was accompanied by a blank containing all the components except L-DOPA. The results were compared with a control consisting of $50 \%$ DMSO replacing the sample. The percentage of tyrosinase inhibition was calculated as follows: [(Acontrol - Asample) / Acontrol] $\times 100$.

\subsection{Microencapsulation of the individual compounds}

\subsubsection{Microencapsulation procedure}

The atomization/coagulation technique was employed to microencapsulate the compounds according to the procedure previously described by Caleja et al. [19], with slight modifications. Sodium alginate, in combination with calcium chloride $\left(\mathrm{CaCl}_{2}\right)$, to promote alginate gelation, was used. The microspheres were produced as follows: $100 \mathrm{mg}$ of the extract was suspended in $20 \mathrm{~mL}$ of distilled water in the presence of $100 \mathrm{mg}$ of tween 80 as surfactant. The mixture was then homogenized for 20 min using a Unidrive X100 homogenizer (Ingenieurbüro CAT, Germany). Thereafter, $400 \mathrm{mg}$ of sodium alginate were added to the suspension, being this mixture kept under stirring until complete dissolution of alginate was achieved. The produced mixture was atomized in a NISCO Var J30 system (Zurich, Switzerland) at a flow rate of $0.3 \mathrm{~mL} / \mathrm{min}$ and a nitrogen pressure of $100 \mathrm{mbar}$, and coagulation performed using $250 \mathrm{~mL}$ of a $\mathrm{CaCl}_{2}$ solution (concentration of $4 \%(\mathrm{w} /$ v)) over a time-period of $4 \mathrm{~h}$. The produced microspheres were then recovered by filtration under reduced pressure, washed twice with distilled water, lyophilized and stored in dark conditions at $4{ }^{\circ} \mathrm{C}$. For easy identification of the samples, the following codes were assigned to each type of microparticles, denoting the contained ingredient: A empty microparticles B - p-hydroxycinnamic acid, C - p-coumaric acid, D - cinnamic acid, E - protocatechuic acid, F - Ergosterol.

\subsubsection{Microencapsulation efficiency, yield and load}

2.3.2.1. Encapsulation efficiency (EE \%). This parameter measures the ability of the encapsulating material to properly encapsulate the bioactive ingredient. It translates the amount effectively microencapsulated, which can be achieved by measuring the amount that has not been encapsulated (in the present case, the cumulative amount of the compound present in the coagulation solution and in the first washing solution), with respect to the theoretical amount used in the formulation (Eq. (1)). Quantification of the respective compound was done by HPLC coupled to a photodiode array detector (PDA) [20].

$\mathrm{EE}(\%)=\frac{\text { weight of the encapsulated bioactive }}{\text { weight of bioactive used in the formulation }} \times 100$

2.3.2.2. Encapsulation yield ( $E Y \%)$. This parameter relates the total amount of produced microcapsules with the amount of atomized material (Eq. (2)).
$\mathrm{EY}(\%)=\frac{\text { weight of produced micropheres }}{\text { weight of the atomized formulation }} \times 100$

2.3.2.3. Encapsulation load (EL \%). This parameter measures the bioactive content $(\%, \mathrm{w} / \mathrm{w})$ present in the produced microspheres and can be calculated using Eq. (3).

$\mathrm{EL}(\%)=\frac{\text { weight of the encapsulated bioactive }}{\text { weight of the produced micropheres }} \times 100$

\subsubsection{Microcapsules characterization}

Microspheres were analysed by optical microscopy (OM) using a Nikon Eclipse 50i microscope (Tokyo, Japan) equipped with a Nikon Digital Sight camera and NIS Elements software for data acquisition. OM analysis was applied to assess the size and morphology of the microspheres right after the atomization and during the coagulation stage. This analysis can be also useful to check the presence of the extract inside the microspheres.

The particle size distributions were determined by laser diffraction using a Malvern Mastersizer 3000 laser equipped with a Hydro MV dispersion unit (Malvern, UK). Data was obtained and treated with Mastersizer software version 3.10. Water was used as the sample dispersion medium. Five measurements were made to ensure reproducibility, and the results were expressed in volume and number, being particle size results expressed as the $10 \%, 50 \%$ and $90 \%$ percentiles. All the residual values were $<1 \%$. The span of the particle size distribution, based on the volume distribution, was also determined using the expression (Dv90 - Dv10) / Dv50 [21].

\subsection{Incorporation of the free and microencapsulated compounds into the cosmetic formulations}

A cosmetic base cream with light colour, free from fragrances, colourants, parabens, mineral oils, sodium lauryl sulphate (SLS), propylene glycol, and ethoxylates, purchased from Fagron Iberica S.A.U. (Barcelona, Spain) was used. According to the technical data available, this formulation has been certified to be safe by the US Food and Drug Administration (FDA), Regulation (EC) No 1907/2006-REACH and the National Health Surveillance Agency (ANVISA) of Brazil. To cover all the evaluated bioactive properties, the base creams were incorporated at a content 2.5 -fold the $\mathrm{EC}_{50}$ and MIC values, which corresponded to a scale of $7 \mathrm{mg}$ of individual compound and $63 \mathrm{mg}$ of microspheres per gram of base cream. A higher incorporation was not viable due to the significant physicochemical changes associated to the adding of the microspheres to the base cream. The individual compounds, or the prepared microspheres, were incorporated into the base cream, with homogenization achieved by stirring. Periodically ( 0 months, 3 months and 6 months), samples were analysed to determine their bioactive properties and physicochemical changes over a 6-month time period. For the determination of the bioactive properties, stock solutions were prepared from the developed formulations by dissolution in a $50 \%$ DMSO solution at a concentration of $100 \mathrm{mg} / \mathrm{mL}$ for anti-tyrosinase and anti-inflammatory activities evaluation. For the antimicrobial activity evaluation, the samples were prepared in $10 \%$ DMSO at $800 \mathrm{mg} / \mathrm{mL}$ and kept at $37^{\circ} \mathrm{C}$ to enable formulations dissolution.

For easy identification of the samples, each formulation was labelled as follows: i) Cosmeceutical formulations incorporated with free individual compounds: CFHA - $p$-hydroxybenzoic acid, CFCoA - p-coumaric acid, CFCA - cinnamic acid, CFPA - protocatechuic acid, CFE ergosterol; ii) cosmeceutical formulations incorporated with microencapsulated compounds: CFEM - empty microspheres (control), CFMHA - microspheres with $p$-hydroxybenzoic acid, CFMCoA - microspheres with $p$-coumaric acid, CFMCA - microspheres with cinnamic acid, CFMPA - microspheres with protocatechuic acid, CFME - 
microspheres with ergosterol. For all the assays a negative control was analysed with base cream (BA).

\subsection{Chemical characterization of the cosmeceutical formulations}

The cream formulations containing the free compounds were extracted with methanol for $30 \mathrm{~min}$, filtered, and dried in a rotary evaporator (Büchi R-210, Flawil, Switzerland). For phenolic acids analysis, the samples were dissolved in a water:methanol mixture $(80: 20 \mathrm{v} / \mathrm{v})$, filtered through a $0.22 \mu \mathrm{m}$ nylon disposable filter and then injected in a Shimadzu 20A series ultra-fast liquid chromatograph (UFLC, Shimadzu Corporation, Kyoto, Japan). For ergosterol analysis, the sample was dissolved in methanol, filtered and further injected in the HPLC-UV (Knauer, Smartline system 1000, Berlin, Germany. The individual compounds were quantified using calibration curves obtained from commercial standards, namely: protocatechuic acid $(\mathrm{y}=164,741 \mathrm{x}$; $\left.R^{2}=0.999\right), \quad p$-hydroxybenzoic acid $\left(\mathrm{y}=113,523 \mathrm{x} ; R^{2}=0.999\right)$, $p$-coumaric acid $\left(\mathrm{y}=433,521 \mathrm{x} ; R^{2}=0.998\right), \quad$ cinnamic acid ( $\left.\mathrm{y}=583,527 \mathrm{x} ; R^{2}=0.998\right)$, ergosterol ( $\left.\mathrm{y}=0.71727 \mathrm{x}, R^{2}=0.996\right)$. Clarity 2.4 Software (DataApex) was employed for data analysis. The results were expressed as $\mu \mathrm{g}$ per $\mathrm{g}$ of cream and $\mathrm{mg}$ per $\mathrm{g}$ of cream for phenolic acids and ergosterol, respectively.

\subsection{Physicochemical parameters of the cosmeceutical formulations}

\subsubsection{Colour measurement}

The colour of cosmeceutical formulations was measured using a colourimeter (model CR-400, Konica Minolta Sensing Inc., Tokyo, Japan) that has been calibrated against a standard white tile [22]. The colour was measured using the $L^{*} a^{*} b^{*}$ system where $L^{*}$ is a measure of the lightness, $a^{*}$ is a measure of the greenness/redness while $b^{*}$ is a measure of the blueness/yellowness. The colour of each sample was measured in three different points, and the average considered the true colour value. Colour space values were registered using the data software "Spectra Magic Nx" (version CM-S100W 2.03.0006).

\subsection{2. $p H$ measurement}

The $\mathrm{pH}$ of cosmeceutical formulations was measured directly, in triplicate, with a HI $99161 \mathrm{pH}$-meter (Hanna Instruments, Woonsocket, Rhode Island, USA) calibrated before each measurement. The $\mathrm{pH}$ readings were done in triplicate to ensure reproducibility.

\subsection{Statistical analysis}

For all the experiments three samples were analysed and all the assays were carried out in triplicate. The results are expressed as mean values \pm standard deviation (SD). The differences between the different samples were analysed using one-way analysis of variance (ANOVA) followed by Tukey's honestly significant difference post hoc test with $\alpha=0.05$, coupled with Welch's statistic. This treatment was carried out using SPSS v. 22.0 program.

\section{Results and discussion}

\subsection{Bioactive properties of the individual compounds}

In the present work, the potential of the studied compounds to suppress NO production was evaluated (Table 1a). Regarding the results, $p$-coumaric acid showed the highest potential presenting the lowest $\mathrm{EC}_{50}$ values $(152.17 \pm 6.22 \mu \mathrm{g} / \mathrm{mL})$, followed by cinnamic $(180.12 \pm 14.18 \mu \mathrm{g} / \mathrm{mL})$, protocatechuic $(182.16 \pm 9.76 \mu \mathrm{g} / \mathrm{mL})$ and p-hydroxybenzoic acids $(195.21 \pm 8.70 \mu \mathrm{g} / \mathrm{mL})$. These results are in agreement with the ones reported previously by our research group for cinnamic and $p$-hydroxybenzoic acids [14]. On the other hand, ergosterol showed lower activity ( $338 \pm 23 \mu \mathrm{g} / \mathrm{mL}$ ), possibly due to its low solubility in the culture medium. To our best knowledge, only Kuo et al.
Table 1a

Anti-inflammatory and anti-tyrosinase activities of the individual compounds.

\begin{tabular}{lcc} 
Individual compounds & $\begin{array}{c}\text { Anti-inflammatory activity } \\
\left(\mathrm{EC}_{50} \text { values, } \mu \mathrm{g} / \mathrm{mL}\right)\end{array}$ & $\begin{array}{c}\text { Anti-tyrosinase activity } \\
\left(\mathrm{EC}_{50} \text { values, } \mathrm{mg} / \mathrm{mL}\right)\end{array}$ \\
\hline Cinnamic acid & $180 \pm 14^{\mathrm{c}}$ & $0.31 \pm 0.05^{*}$ \\
Protocatechuic acid & $182 \pm 10^{\mathrm{c}}$ & $>2.0$ \\
$p$-Hydroxybenzoic acid & $195 \pm 9^{\mathrm{b}}$ & $1.86 \pm 0.01^{*}$ \\
$p$-Coumaric acid & $152 \pm 6^{\mathrm{d}}$ & $>2.0$ \\
Ergosterol & $338 \pm 23^{\mathrm{a}}$ & $>2.0$ \\
\hline
\end{tabular}

Anti-inflammatory activity is expressed as $\mathrm{EC}_{50}$ values corresponding to $50 \%$ of inhibition of the NO production in comparison with the negative control $(100 \%$ of NO production). Dexamethasone (positive control) $\mathrm{EC}_{50}$ values: $16 \mu \mathrm{g} / \mathrm{mL}$. Anti-tyrosinase activity is expressed as $\mathrm{EC}_{50}$ values corresponding to $50 \%$ of inhibition of tyrosinase. Ascorbic acid (positive control) $\mathrm{EC}_{50}$ values: $31 \mu \mathrm{g} / \mathrm{mL}$. In the column different letters mean significant differences $(p<0.05)$.

* Statistically different values, Student's $t$-test $p$-value $<0.001$.

[23] have reported an anti-inflammatory potential for commercial ergosterol at $10 \mu \mathrm{g} / \mathrm{mL}$ by inhibiting NO production in RAW. 7 macrophage cells.

Concerning the anti-tyrosinase activity (Table 1a), cinnamic acid displayed the strongest activity exhibiting the lowest $\mathrm{EC}_{50}$ value $(0.31 \pm 0.05 \mathrm{mg} / \mathrm{mL})$. The other tested compounds, showing $\mathrm{EC}_{50}$ values above the highest tested concentration of $2.0 \mathrm{mg} / \mathrm{mL}$, presented limited activity. To the author's best knowledge, no studies have been previously reported for the tyrosinase inhibitory activity of protocatechuic, $p$-hydroxybenzoic and cinnamic acids; however, some other phenolic acids such as caffeic acid $(43.09 \pm 2.3 \mu \mathrm{M})$, ferulic acid $(51.85 \pm 1.7 \mu \mathrm{M})$ and gallic acid $(18.30 \mu \mathrm{M})$ have been reported as significantly suppress tyrosinase activity $[7,24]$. Although in the present study it was not possible to report anti-tyrosinase activity for $p$ coumaric acid, studies conducted by An et al. [25] related that p-coumaric acid at $10 \mu \mathrm{g} / \mathrm{mL}$ showed activity at levels comparable to kojic acid, whereas Jun et al. [26] confirmed that the anti-tyrosinase activity mechanism of $p$-coumaric acid was due to its ability to reduce microphthalmia-associated transcription factor (MITF) and tyrosinase mRNA expression by 73 and $82 \%$, respectively. The results show that these compounds display a promising potential to be used, either alone or in combination with other bioactive ingredients, to provide protection against hyperpigmentation.

The results for the antimicrobial activity expressed as MIC values are shown in Table 1b for the studied compounds. All the individual compounds showed considerable antimicrobial capacity against gram positive, gram negative bacteria and Candida, with cinnamic acid and ergosterol exhibiting a similar antimicrobial pattern. Interestingly, $p$ coumaric and protocatechuic acids presented the highest activity against methicillin sensitive Staphylococcus aureus (SA), similarly to what was reported by Parkar et al. [27]. All the compounds showed inhibitory activity against $E$. coli in accordance with the results reported by Cueva et al. [28]. Due to the well-known constraints associated with the use of synthetic antimicrobial agents, this study highlights the potential of the studied polyphenols and sterols as viable alternatives. Phenolic acids such as ferulic and caffeic acid have been reported to be interesting anti-wrinkling and photoprotective ingredients for skin care products, as reported by Pluemsamran et al. [29], while Seok and Boo [30] have reported anti-ageing potential for $p$-coumaric acid in dermal fibroblasts cell lines. However, most of the cosmeceutical properties were only evaluated for the individual compounds, and the compounds seldom incorporated in base creams to evaluate if the bioactive properties are maintained.

\subsection{Morphology and characteristics of the developed microspheres}

The surface morphology of the prepared microspheres, visualised by optical microscopy, right after preparation, and at magnifications of 
Table 1b

Antimicrobial activity of the individual compounds.

\begin{tabular}{|c|c|c|c|c|c|c|}
\hline & \multirow[t]{2}{*}{ Bacterial strains } & \multicolumn{5}{|l|}{$\mathrm{MIC}(\mathrm{mg} / \mathrm{mL})$} \\
\hline & & Cinnamic acid & $p$-Hydroxybenzoic acid & $p$-Coumaric acid & Protocatechuic acid & Ergosterol \\
\hline \multirow[t]{3}{*}{ Gram+ } & MRSA & 1 & 1 & 0.5 & 2.5 & 1 \\
\hline & MSSA & 1 & 1 & 0.5 & 2.5 & 1 \\
\hline & E. faecalis & 1 & 1 & 1 & 1 & 1 \\
\hline \multirow[t]{4}{*}{ Gram- } & E. coli & 1 & 1 & 2.5 & 0.25 & 1 \\
\hline & P. mirabilis & 1 & 2.5 & 2.5 & 2.5 & 1 \\
\hline & Yeast strain & & & & & \\
\hline & C. albicans & 2.5 & 2.5 & 2.5 & 2.5 & 2.5 \\
\hline
\end{tabular}

$\mathbf{a}$ $40 \mathrm{x}$

$100 x$

$400 \mathrm{x}$

100x lyophylised

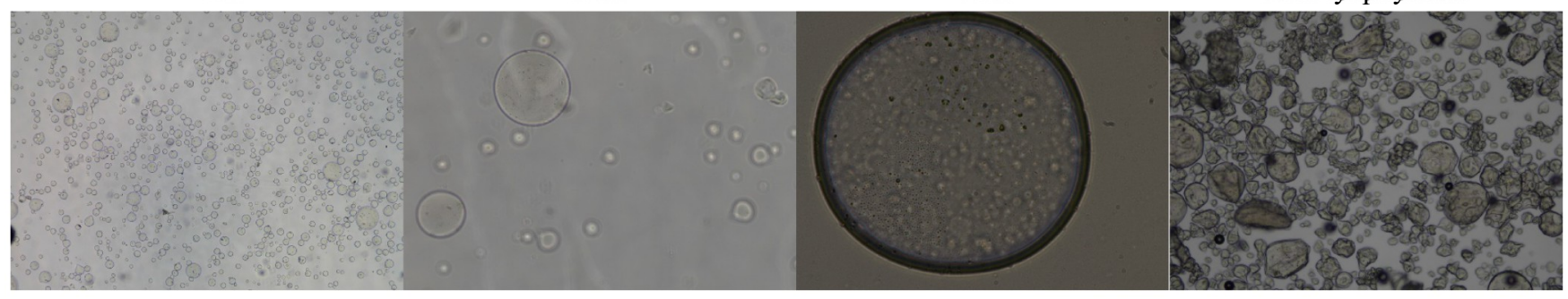

b
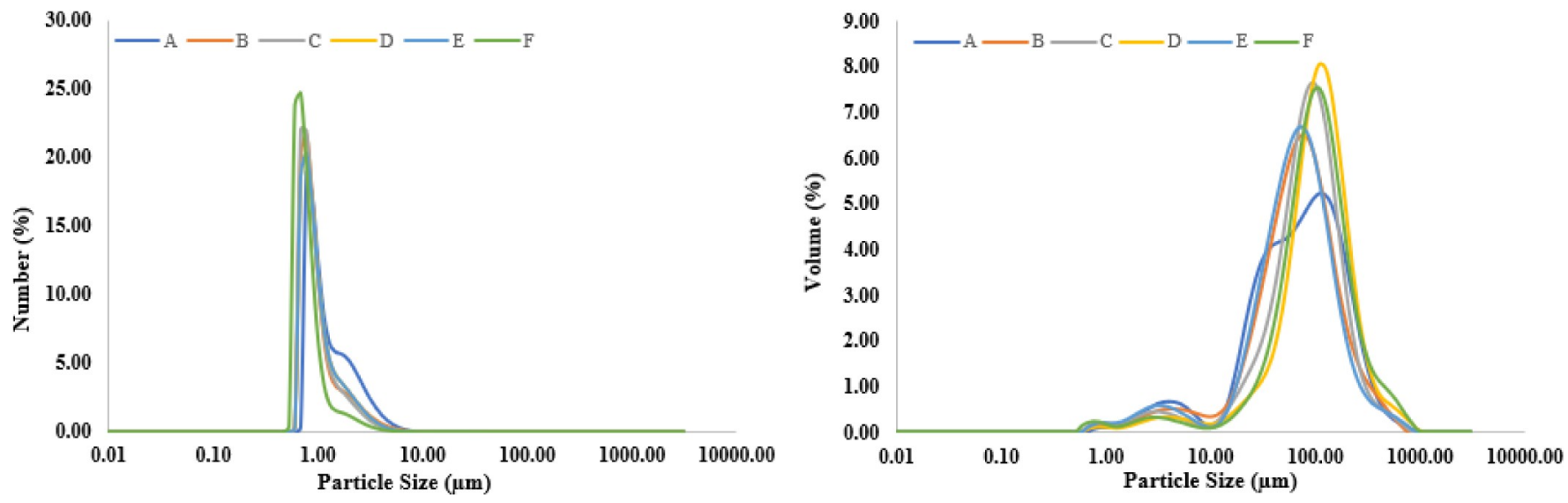

Fig. 1. a. Optical microscopy analysis with magnifications of 40,100 and $400 \times$ of the microspheres after $4 \mathrm{~h}$ of coagulation under stirring. b. Microparticles size distribution in number and volume.

$40 \times, 100 \times$ and $400 \times$, is shown in Fig. 1a. It is possible to observe a consistent spherical morphology with particles of various sizes showing no agglomeration. The lyophilized microspheres were rehydrated in distilled water and analysed using the optical microscope at a magnification of $100 \times$. The final shape of the rehydrated microspheres was quite irregular, but close to what was observed with microspheres right after the encapsulation process.

The results for the particle size distribution, in number and volume, represented as percentiles (D10, D50 and D90), are presented in Table 2. From the analysis of particle size distribution (Fig. 1b), unimodal distributions in number can be depicted, whereas the distributions in volume were bimodal. Another important parameter to consider is the Span (dispersity) which gives an indication of the particle size distribution width and gives an indication of the dispersion of the particle size distribution, i.e. of the size homogeneity of the produced microparticles. From the obtained data, there is no significant dissimilarity among the particles incorporating the various bioactive ingredients; only the empty microspheres showed a significant variation (higher particle size distribution).
Table 2 summarizes the results obtained for the encapsulation yield, loading, and efficiency of the produced alginate microspheres. To determine $\mathrm{EE} \%$, the concentration of the analysed compounds was determined both in the coagulation and washing solutions, and the amount effectively encapsulated obtained by difference. The results showed that the compounds were successfully encapsulated with EE $\%>50 \%$ for all the studied cases. The EY \%, which represents the weight of dry microspheres, recovered after encapsulation process, relatively to the weight of raw-materials initially use, was found to be over $45 \%$ in all the samples. These values are quite satisfactory considering that the applied technique was conducted at a laboratory scale. Some authors reported the encapsulation of phenolic acids such as caffeic, chlorogenic and rosmarinic acids, using similar scales, with a EY \% only over $37 \%$, for all the studied cases [31]. The EL\%, representing the compound content per gram of microspheres, allows the determination of the microsphere's amount needed to be incorporated in the semi solid base creams. By analysing the results listed in Table 2, the EL \% was above $5 \%$ in all the cases. 
Table 2

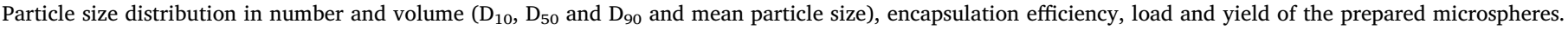

\begin{tabular}{|c|c|c|c|c|c|c|c|c|c|c|c|c|}
\hline \multirow[t]{3}{*}{ Samples } & \multirow[t]{3}{*}{$\mathrm{EE}(\%)$} & \multirow[t]{3}{*}{ EY (\%) } & \multirow[t]{3}{*}{ EL (\%) } & \multicolumn{7}{|c|}{ Particle size distribution $(\mu \mathrm{m})$} & \multicolumn{2}{|c|}{ Mean particle size $(\mu \mathrm{m})$} \\
\hline & & & & \multicolumn{4}{|c|}{ Volume distribution } & \multicolumn{3}{|c|}{ Number distribution } & \multirow[t]{2}{*}{ Number } & \multirow[t]{2}{*}{ Volume } \\
\hline & & & & $\mathrm{D}_{10}$ & $\mathrm{D}_{50}$ & $\mathrm{D}_{90}$ & Span & $\mathrm{D}_{10}$ & $\mathrm{D}_{50}$ & $\mathrm{D}_{90}$ & & \\
\hline A & na & 60.2 & na & 19.5 & 79.2 & 235 & 2.72 & 0.82 & 1.15 & 2.83 & 1.50 & 102 \\
\hline B & 88.3 & 57.8 & 9.80 & 20.5 & 74.6 & 206 & 2.28 & 0.72 & 0.93 & 2.10 & 1.63 & 94.5 \\
\hline C & 73.7 & 55.7 & 8.19 & 25.4 & 90.7 & 208 & 2.01 & 0.71 & 0.91 & 1.84 & 1.07 & 104 \\
\hline $\mathrm{D}$ & 98.4 & 58.9 & 10.9 & 36.1 & 115 & 261 & 1.95 & 0.72 & 0.91 & 1.95 & 1.10 & 132 \\
\hline E & 50.3 & 62.2 & 5.59 & 21.3 & 71.0 & 186 & 2.31 & 0.72 & 0.95 & 2.05 & 1.15 & 90.0 \\
\hline $\mathrm{F}$ & 63.2 & 45.5 & 7.02 & 36.3 & 108 & 273 & 2.19 & 0.63 & 0.77 & 1.26 & 0.86 & 132 \\
\hline
\end{tabular}

na-not applied; A - empty microparticles B - p-hydroxycinnamic acid, C - p-coumaric acid, D - cinnamic acid, E - protocatechuic acid, F - ergosterol.

\subsection{Bioactive properties of cosmeceutical formulations}

In the present work, the cosmeceutical potential of each prepared formulation was investigated based on their ability to display anti-inflammatory, anti-tyrosinase and antimicrobial activities. The results for the in vitro anti-tyrosinase activity of the prepared cosmeceutical formulations containing the individual compounds are presented in Table 2. From the results, all the developed formulations (CFHA, CFCoA, CFCA, CFPA) were able to inhibit tyrosinase activity over 6 months under storage. On the other hand, the formulation prepared with ergosterol (CFE) was also able to inhibit the tyrosinase activity, however, a significant loss was observed as the storage time increased, which can be attributed to the interaction between ergosterol and other ingredients present in the base cream (Table 3a). Several studies have reported that phenolic acids and related compounds are the most significant contributors to anti-tyrosinase activity, but most of these studies only investigated pure compounds without perform their incorporation in topical semi-solid formulations [3,32]. An exception was the work of Seo et al. [33], who conducted clinical trials by exposing the forearms of 21 persons after topical application of a $p$-coumaric acid-based cream twice daily. The results showed that there was a significant improvement in the hyperpigmentation formation.

For the anti-inflammatory activity, all the developed formulations containing the individual phenolic acids, cinnamic acid and ergosterol were able to inhibit NO production (Table 3a). As observed for the other bioactive properties there was a gradual loss of bioactivity as the storage time increased, with the ergosterol-based formulation (CFE) showing the lower anti-inflammatory activity. The formulation prepared with $p$-coumaric acid ( $\mathrm{CFCoA}$ ) was the most promising one, as determined based on the observations made with the individual compounds.

The antimicrobial activity of the developed cosmeceutical formulations, against gram negative, gram positive and yeast microbial strains, are presented in Tables $3 \mathrm{a}$ and $3 \mathrm{~b}$. In all the cases, the prepared formulations were found to be effective against Gram negative, Gram positive bacteria and $C$. albicans. The antimicrobial activity of the formulations incorporated with the free individual agents decreased along the storage time, probably due to the interaction with the ingredients present in the base cream. Our findings suggest that these bioactive compounds have the potential to be used as preservatives capable of preventing microbial contamination in cosmeceutical formulations.

The identification and quantification of the individual compounds present in the prepared formulations, determined by HPLC, are presented in Table 3 a. It can be concluded that all the target compounds were stable and where identified in their corresponding base cream formulation. The validation of an extraction protocol for phenolic acids extraction from semi-solid formulation still poses some challenges. However, it was possible to effectively identify and quantify the amount of phenolic acids and related compounds present in each prepared cosmeceutical formulation over the storage time. The results showed that there was no significant loss of phenolic compounds, or ergosterol, over the course of incubation period for all the considered semi-solid formulations.

Table 3a

Bioactive properties of the individual compounds, developed cosmeceutical formulations and compounds monitoring over the storage time.

\begin{tabular}{|c|c|c|c|c|c|c|c|c|c|}
\hline \multirow[t]{2}{*}{ Creams } & \multicolumn{3}{|c|}{ Anti-tyrosinase activity ( $\mathrm{EC}_{50}$ values, $\mathrm{mg} / \mathrm{mL}$ ) } & \multicolumn{3}{|c|}{ Anti-inflammatory activity ( $\mathrm{EC}_{50}$ values, $\mathrm{mg} / \mathrm{mL}$ ) } & \multicolumn{3}{|c|}{ Bioactive compounds profile (mg/g) } \\
\hline & Time 0 & Time 3 & Time 6 & Time 0 & Time 3 & Time 6 & Time 0 & Time 3 & Time 6 \\
\hline CFHA & $54 \pm 4^{\mathrm{b}}$ & $55.3 \pm 0.1^{b}$ & $59.9 \pm 0.5^{\mathrm{a}}$ & $30.66 \pm 1^{\mathrm{c}}$ & $41.92 \pm 2.17^{b}$ & $54.13 \pm 0.93^{\mathrm{a}}$ & $6.80 \pm 0.07^{\mathrm{a}}$ & $6.83 \pm 0.04^{\mathrm{a}}$ & $6.39 \pm 0.02^{\mathrm{b}}$ \\
\hline CFCoA & $37.6 \pm 0.9^{b}$ & $41 \pm 7^{b}$ & $51 \pm 6^{\mathrm{a}}$ & $22.64 \pm 0.76^{c}$ & $33.04 \pm 1.51^{b}$ & $45.29 \pm 1.63^{\mathrm{a}}$ & $6.67 \pm 0.03^{\mathrm{a}}$ & $6.52 \pm 0.08^{b}$ & $6.48 \pm 0.07^{b}$ \\
\hline CFCA & $32.3 \pm 0.2^{\mathrm{b}}$ & $36 \pm 5^{\mathrm{ab}}$ & $40 \pm 3^{\mathrm{a}}$ & $33.47 \pm 0.46^{\mathrm{c}}$ & $40.03 \pm 1.09^{b}$ & $47.05 \pm 1.01^{\mathrm{a}}$ & $6.50 \pm 0.01^{\mathrm{a}}$ & $6.4 \pm 0.2^{\mathrm{a}}$ & $6.39 \pm 0.02^{\mathrm{a}}$ \\
\hline CFPA & $40 \pm 3^{\mathrm{b}}$ & $43 \pm 2^{b}$ & $61 \pm 7^{\mathrm{a}}$ & $36.28 \pm 1.62$ & $47.59 \pm 0.55$ & $>60$ & $6.64 \pm 0.08^{\mathrm{a}}$ & $6.5 \pm 0.1^{\mathrm{b}}$ & $6.42 \pm 0.01^{\mathrm{b}}$ \\
\hline $\mathrm{CFE}$ & $24 \pm 2^{c}$ & $51 \pm 5^{b}$ & $98.2 \pm 0.7^{\mathrm{a}}$ & $53.35 \pm 1.62$ & $>60$ & $>60$ & $6.9 \pm 0.01^{\mathrm{a}}$ & $6.81 \pm 0.01^{\mathrm{b}}$ & $6.78 \pm 0.02^{b}$ \\
\hline CFEM & $>100$ & $>100$ & $>100$ & $>60$ & $>60$ & $>60$ & nd & nd & nd \\
\hline CFMHA & $>100$ & $>100$ & $48 \pm 12$ & $>60$ & $50.56 \pm 0.57$ & $42.66 \pm 0.94$ & nd & nd & nd \\
\hline CFMCoA & $>100$ & $73 \pm 1^{*}$ & $45 \pm 4$ & $>60$ & $42.34 \pm 1.02$ & $34.57 \pm 0.43^{*}$ & nd & nd & nd \\
\hline CFMCA & $>100$ & $80.8 \pm 0.7^{*}$ & $43.0 \pm 0.8$ & $>60$ & $48.440 .5 \pm \pm^{*}$ & $40.33 \pm 1.13^{*}$ & nd & nd & nd \\
\hline CFMPA & $>100$ & $>100$ & $49 \pm 8$ & $>60$ & $46.99 \pm 1.43^{*}$ & $40.07 \pm 0.17^{*}$ & nd & nd & nd \\
\hline CFME & $>100$ & $47 \pm 4$ & $32.6 \pm 0.6^{*}$ & $>60$ & $>60$ & $>60$ & nd & nd & nd \\
\hline BA & $>100$ & $>100$ & $>100$ & $>60$ & $>60$ & $>60$ & nd & nd & nd \\
\hline
\end{tabular}

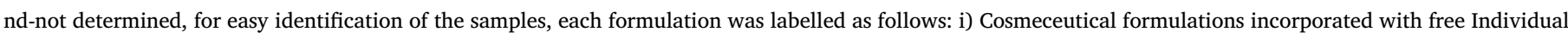

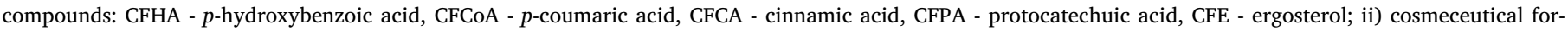

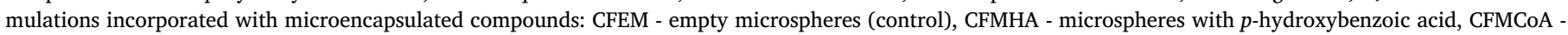

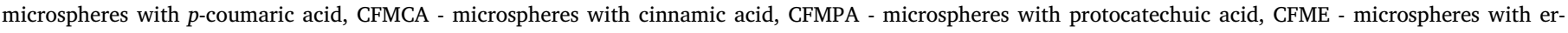
gosterol. For all the assays a negative control was analysed with base cream (BA). In each row different letters mean significant differences $(p<0.05)$.

* Statistically different values, Student's $t$-test $p$-value $<0.001$. 
Table 3b

Antimicrobial activity of the final cosmeceutical formulations.

\begin{tabular}{|c|c|c|c|c|c|c|c|c|c|c|c|c|c|c|c|c|c|c|}
\hline \multirow[t]{4}{*}{ Samples } & \multicolumn{18}{|c|}{ Mic $(\mathrm{mg} / \mathrm{mL})$} \\
\hline & \multicolumn{15}{|c|}{ Bacterial strains } & \multirow{2}{*}{\multicolumn{3}{|c|}{$\frac{\text { Yeast }}{\text { C. albicans }}$}} \\
\hline & \multicolumn{3}{|c|}{ E. faecalis } & \multicolumn{3}{|l|}{ MSSA } & \multicolumn{3}{|l|}{ MRSA } & \multicolumn{3}{|l|}{ E. coli } & \multicolumn{3}{|c|}{ P. mirabilis } & & & \\
\hline & $\mathrm{T}_{0}$ & $\mathrm{~T}_{3}$ & $\mathrm{~T}_{6}$ & $\mathrm{~T}_{0}$ & $\mathrm{~T}_{3}$ & $\mathrm{~T}_{6}$ & $\mathrm{~T}_{0}$ & $\mathrm{~T}_{3}$ & $\mathrm{~T}_{6}$ & $\mathrm{~T}_{0}$ & $\mathrm{~T}_{3}$ & $\mathrm{~T}_{6}$ & $\mathrm{~T}_{0}$ & $\mathrm{~T}_{3}$ & $\mathrm{~T}_{6}$ & $\mathrm{~T}_{0}$ & $\mathrm{~T}_{3}$ & $\mathrm{~T}_{6}$ \\
\hline BA & $>400$ & $>400$ & $>400$ & $>400$ & $>400$ & $>400$ & $>400$ & $>400$ & $>400$ & $>400$ & $>400$ & $>400$ & $>400$ & $>400$ & $>400$ & $>400$ & $>400$ & $>400$ \\
\hline CFHA & 200 & 200 & 400 & 200 & 200 & 400 & 200 & 200 & 400 & 200 & 200 & 400 & 400 & 400 & 400 & 400 & 400 & 400 \\
\hline CFCoA & 200 & 200 & 400 & 100 & 100 & 200 & 100 & 100 & 200 & 400 & 400 & 400 & 200 & 200 & 400 & 400 & 400 & 400 \\
\hline CFCA & 200 & 200 & 400 & 200 & 200 & 400 & 200 & 200 & 400 & 200 & 200 & 400 & 200 & 200 & 400 & 400 & 400 & 400 \\
\hline CFPA & 200 & 200 & 400 & 100 & 100 & 200 & 100 & 100 & 200 & 400 & 400 & 400 & 400 & 400 & 400 & 400 & 400 & 400 \\
\hline CFE & 200 & 200 & 400 & 200 & 200 & 400 & 200 & 200 & 400 & 200 & 200 & 400 & 200 & 200 & 400 & 400 & 400 & 400 \\
\hline CFEM & $>400$ & $>400$ & $>400$ & $>400$ & 400 & $>400$ & $>400$ & $>400$ & $>400$ & $>400$ & $>400$ & $>400$ & $>400$ & $>400$ & $>400$ & $>400$ & $>400$ & $>400$ \\
\hline CFMHA & $>400$ & 400 & 200 & $>400$ & 400 & 200 & $>400$ & 400 & 200 & $>400$ & 400 & 200 & $>400$ & $>400$ & 400 & $>400$ & $>400$ & 400 \\
\hline CFMCoA & $>400$ & 400 & 200 & $>400$ & 200 & $>400$ & $>400$ & 200 & 100 & $>400$ & $>400$ & 400 & $>400$ & $>400$ & 200 & $>400$ & $>400$ & $>400$ \\
\hline CFMCA & $>400$ & 400 & 200 & $>400$ & 400 & 200 & $>400$ & 400 & 200 & $>400$ & $>400$ & 400 & $>400$ & $>400$ & 400 & $>400$ & $>400$ & $>400$ \\
\hline CFMPA & $>400$ & 400 & 200 & $>400$ & 200 & 100 & $>400$ & 200 & 100 & $>400$ & $>400$ & 400 & $>400$ & $>400$ & 400 & $>400$ & $>400$ & $>400$ \\
\hline CFME & $>400$ & 400 & 200 & $>400$ & 400 & 200 & $>400$ & 400 & 200 & $>400$ & $>400$ & 400 & $>400$ & $>400$ & 400 & $>400$ & $>400$ & 400 \\
\hline
\end{tabular}

\subsection{Real time in vitro release}

Real time in vitro release of the bioactive compounds from a polymeric microsphere is typically conducted to evaluate the control release at appropriate environmental and physiological conditions. Sometimes the release may take weeks, months, or even years [34]. The real time in vitro release of the microencapsulated bioactive agents was performed under controlled temperature medium. Their release was evaluated based on their potential to increase the bioactive properties of the corresponding formulations. From the obtained data, microspheres showed a very slow release of the encapsulated bioactives up to the 6th month under storage. Throughout the process of microencapsulation, the anti-tyrosinase, anti-inflammatory and antimicrobial activities were not compromised since the bioactivity was kept in almost all cases, highlighting the potential of alginate particles as effective delivery vehicles. Regarding the anti-tyrosinase activity, a fast release, sufficient to cause activity, was achieved for the formulations at the 3rd month of storage as shown by the data of Tables $3 \mathrm{a}$ and $3 \mathrm{~b}$. It was also observed that both formulations CFMCoA and CFMCA showed similar profile relative to the anti-tyrosinase and anti-inflammatory activity. The release in both cases was able to sustain the bioactivities up to the 6th month. As expected BCEM did not show any contribution to the tested bioactivities. CFMPA and CFMHA were able to maintain an anti-tyrosinase activity lower than the one obtained for CFMCoA and CFMCA, regardless they showed the best anti-inflammatory activity. BCE was able to sustain the anti-tyrosinase activity, however no anti-inflammatory activity was perceived. The slow release supports the suitability of the microencapsulation process to be used in the design of cosmeceutical formulations, whose shelf life span from months to even years, making the gradual release adequate to ensure the continuous effectiveness against microbial growth. It is also interesting to note that the compounds not released during the evaluated storage time can be further released in the skin during the application of the cosmeceutical formulation. In fact, due to the applied pressure, capsules breakage will occur with subsequent release of the encapsulated compounds, which will result in skin benefits derived from the fraction of preserved bioactives. From the obtained results, the alginate microcapsules showed potential to ensure controlled release of the bioactive agents, which will add in the cosmetic preservation during storage, and will provide benefits for the skin upon application (preserved fraction).

Some relevant reports have been presented on the use of microencapsulation techniques to protect bioactive compounds for cosmeceutical purposes. Biswick et al. [35], microencapsulated caffeic acid, with the produced microparticles able to withstand both UVA and UVB radiation, indicating their suitability to be used in the design of sunscreen cosmetic formulations. Ouimet et al. [36] microencapsulated ferulic acid, whose slow release was reported as acting for days, but able to achieve antioxidant activity. Different factors such as polymer nature and molecular weight, and microparticle's size have been reported by Sharma et al. [37], as influencing the release rate making these issues worth of further investigation. Some studies report an accelerated release under different conditions such as temperature, solvent, $\mathrm{pH}$, enzymes effect or even due to stirring rate [38]. However, some of these conditions do not mimic the real environment, thus the real release mechanism, making the studies performed under the context of the present work valuable tools to access the long-lasting effect of the encapsulated bioactive compounds. The overall results show that microencapsulation can provide efficient tools to support the development of cosmetic formulations promoting the preservation of the cosmeceutical ingredients, and the sustained release of the compounds (i.e. bioactivity maintenance).

\subsection{Physicochemical parameters}

Although it is possible to find in the market, a wide range of cosmetic colours, the majority of consumers still prefer products with the basic white colour. Hence, the cosmetic industry has paid attention to colour management during the design of new products. Responding to this requisite, the physicochemical evaluation of the prepared semisolid formulations in terms of colour, and also $\mathrm{pH}$, was carried out during the storage period at time 0,3 and 6 months (Table 4). Over the course of the six-month incubation period, no significant colour changes were observed, suggesting that the individual compounds are stable, and their degradation does not lead to end products causing significant colour changes. It was possible to observe that the formulations presented lower levels of lightness $\left(l^{*}\right)$ as the storage time increase, which may be derived from the oxidation of the incorporated compounds. Lower $l^{*}$ values and higher yellowness $\left(b^{*}\right)$ was observed for the formulations prepared with the individual compounds, whereas the colour coordinates for the formulations prepared with the microspheres revealed a deeper red (greater $a^{*}$ value), mainly due to the contribution of alginate. Overall, the initial odour (qualitative evaluation) and colour were maintained, i.e. they were not significantly different over the course of the storage time, which are crucial parameters for the preliminary analysis of stability.

The rate of dermal absorption of topical formulations, such as lotions and creams, are often influenced by the product $\mathrm{pH}$. The skin surface $\mathrm{pH}$ is around 5.5, and at this condition, the skin micro-flora, barrier homeostasis and stratum corneum are maintained [39,40]. Several reports have suggested that a $\mathrm{pH}$ between 3.5 and 6.5 can be 
considered suitable for cosmetic formulations since most of the pathogenic bacteria, such as $S$. aureus, thrive best at neutral $\mathrm{pH}$ levels [39]. In this work, all the developed cosmeceutical formulations presented a $\mathrm{pH}$ in the range 3.4-6.0, suggesting that they have a favourable $\mathrm{pH}$ along the storage time. The samples, stored at $4{ }^{\circ} \mathrm{C}$ in darkness, presented no significant $\mathrm{pH}$ variation, which is a clear indication of their stability. Also, most of the products exhibited excellent stability under this temperature.

\section{Conclusion}

Several bioactive compounds have shown relevant cosmeceutical properties, essential to maintain skin health. In this context, developing delivery systems to allow their sustained release is a strategy of high importance in order to take full advantage of their benefits. The findings achieved in this work reveal that the studied compounds ( $p$ hydroxybenzoic, $p$-coumaric, protocatechuic and cinnamic acids, and ergosterol) displayed anti-tyrosinase, antimicrobial and anti-inflammatory activities making them suitable to be explored as multifunctional cosmeceutical ingredients. The atomization/coagulation technique was successfully applied to encapsulate the bioactive agents, conducting to EE\% values above $50 \%$ for all the tested compounds. After incorporating the individual compounds in the cosmetic formulations, bioactive properties were only partially maintained while their microencapsulated formulations have shown a sustained release able to better preserve bioactivity. Moreover, over the course of storage time, no significant changes were observed in the tested stability parameters, namely colour and $\mathrm{pH}$. By the obtained results, the analysed bioactive agents revealed promising bioactivity and stability. Studies related with the in vitro skin permeation using cellbased models to determine topical bioavailability and confirm the absence of cytotoxic effects in fibroblast or keratinocyte cell lines, will the next steps going forward.

\section{Acknowledgements}

The authors are grateful to the Foundation for Science and Technology (FCT, Portugal) and FEDER under Programme PT2020 for financial support to CIMO (UID/AGR/00690/2019) and Associate Laboratory LSRE-LCM - UID/EQU/50020/2019 - funded by national funds through FCT/MCTES (PIDDAC). The authors are also grateful to FCT for S. Heleno's (SFRH/BPD/101413/2014) grants, and L. Barros' contract. The authors are grateful to FEDER-Interreg España-Portugal programme for financial support through the project 0377_Iberphenol_6_E. This work was also funded by the European Structural and Investment Funds (FEEI) through the Regional Operational Program North 2020, within the scope of Project Mobilizador ValorNatural ${ }^{\oplus}$. This work is also funded by the European Agricultural Fund for Rural Development (EAFRD), through the Rural Development Program (PDR2020), within the scope of Project MicoCoating (PDR2020-101-031472).

\section{Conflicts of interest}

No conflict of interest.

\section{Appendix A. Supplementary data}

Supplementary data to this article can be found online at https:// doi.org/10.1016/j.microc.2019.03.059.

\section{References}

[1] J. Arct, K. Pytkowska, Flavonoids as components of biologically active cosmeceuticals, Clin. Dermatol. 26 (2008) 347-357.

[2] O. Taofiq, A.M. González-Paramás, A. Martins, M.F. Barreiro, I.C.F.R. Ferreira, 
Mushrooms extracts and compounds in cosmetics, cosmeceuticals and nutricosmetics-a review, Ind. Crop. Prod. 90 (2016) 38-48.

[3] O. Taofiq, A.M. González-paramás, M.F. Barreiro, I.C.F.R. Ferreira, Hydroxycinnamic acids and their derivatives: cosmeceutical significance, challenges and future perspectives, a review, Molecules. 22 (2017) 1-24.

[4] H. Masaki, Role of antioxidants in the skin: anti-aging effects, J. Dermatol. Sci. 58 (2010) 85-90.

[5] M.R. Barroso, L. Barros, M. Dueñas, A.M. Carvalho, C. Santos-Buelga, I.P. Fernandes, M.F. Barreiro, I.C.F.R. Ferreira, Exploring the antioxidant potential of Helichrysum stoechas (L.) Moench phenolic compounds for cosmetic applications: chemical characterization, microencapsulation and incorporation into a moisturizer, Ind. Crops Prod. 53 (2014) 330-336.

[6] S.A. Heleno, A. Martins, M.J.R.P. Queiroz, I.C.F.R. Ferreira, Bioactivity of phenolic acids: metabolites versus parent compounds: a review, Food Chem. 173 (2015) 501-513.

[7] K.J.S. Kumar, M.G. Vani, S.Y. Wang, J.W. Liao, L.S. Hsu, H.L. Yang, Y.C. Hseu, In vitro and in vivo studies disclosed the depigmenting effects of gallic acid: a novel skin lightening agent for hyperpigmentary skin diseases, BioFactors. 39 (2013) 259-270.

[8] J.H. Kang, J.E. Jang, S.K. Mishra, H.J. Lee, C.W. Nho, D. Shin, M. Jin, M.K. Kim, C. Choi, S.H. Oh, Ergosterol peroxide from Chaga mushroom (Inonotus obliquus) exhibits anti-cancer activity by down-regulation of the $\beta$-catenin pathway in colorectal cancer, J. Ethnopharmacol. 173 (2015) 303-312.

[9] S. Kim, S. Park, T. Min, K. Yu, Antioxidant activity of ergosterol peroxide (5,8epidioxy-5,8-ergosta-6,22E-dien-3 -ol) in Armillariella mellea, Bull. Kor. Chem. Soc. 20 (1999) 819-823.

[10] O. Taofiq, A. Martins, M.F. Barreiro, I.C.F.R. Ferreira, Anti-inflammatory potential of mushroom extracts and isolated metabolites, Trends Food Sci. Technol. 50 (2016) 193-210.

[11] Z.F. Yan, Y. Yang, F.H. Tian, X.X. Mao, Y. Li, C.T. Li, Inhibitory and acceleratory effects of Inonotus obliquus on tyrosinase activity and melanin formation in b16 melanoma cells, Evidence-Based Complement. Altern. Med. 259836 (2014) 1-11.

[12] L.F. Eichenfield, W.L. Tom, T.G. Berger, A. Krol, A.S. Paller, K. Schwarzenberger, J. N. Bergman, S.L. Chamlin, D.E. Cohen, K.D. Cooper, K.M. Cordoro, D.M. Davis, S.R. Feldman, J.M. Hanifin, D.J. Margolis, R.A. Silverman, E.L. Simpson, H.C. Williams, C.A. Elmets, J. Block, C.G. Harrod, W.S. Begolka, R. Sidbury, Guidelines of care for the management of atopic dermatitis: section 2. Management and treatment of atopic dermatitis with topical therapies, J. Am. Acad. Dermatol. 71 (2014) 116-132.

[13] S.C.-Y. Lin, Y. Wang, D.F. Wertheim, A.G.A. Coombes, Production and in vitro evaluation of macroporous, cell-encapsulating alginate fibres for nerve repair, Mater. Sci. Eng. C. 73 (2017) 653-664.

[14] O. Taofiq, R.C. Calhelha, S. Heleno, L. Barros, A. Martins, C. Santos-Buelga, M.J.R.P. Queiroz, I.C.F.R. Ferreira, The contribution of phenolic acids to the antiinflammatory activity of mushrooms: screening in phenolic extracts, individual parent molecules and synthesized glucuronated and methylated derivatives, Food Res. Int. 76 (2015) 821-827.

[15] CLSI (Ed.), Performance Standards for Antimicrobial Susceptibility Testing. 27th Ed. CLSI Supplement M100, Wayne, PA, Clinical and Laboratory Standards Institute, 2017.

[16] Sociéte Francaise de Microbiologie, Comité de L'Antibiogramme de la Société Française de Microbiologie; Communiqué; Société Française de Microbiologie:, Com. L'Antibio. 2012 (2013).

[17] EUCAST, European Committee on Antimicrobial Susceptibility Testing (EUCAST); European Society of Clinical Microbiology and Infectious Diseases (ESCMID), Stock. Sweden. (2013).

[18] O. Taofiq, S. Heleno, R. Calhelha, M. Alves, L. Barros, M. Barreiro, A. GonzálezParamás, I. Ferreira, Development of mushroom-based cosmeceutical formulations with anti-inflammatory, anti-tyrosinase, antioxidant, and antibacterial properties, Molecules. 21 (2016) 1-12.

[19] C. Caleja, A. Ribeiro, L. Barros, J.C.M. Barreira, A.L. Antonio, M.B.P.P. Oliveira, M.F. Barreiro, I.C.F.R. Ferreira, Cottage cheeses functionalized with fennel and chamomile extracts: comparative performance between free and microencapsulated forms, Food Chem. 199 (2016) 720-726.
[20] S. Papadimitriou, D. Bikiaris, Novel self-assembled core-shell nanoparticles based on crystalline amorphous moieties of aliphatic copolyesters for efficient controlled drug release, J. Control. Release 138 (2009) 177-184.

[21] F.M. Barboza, W.M. Machado, L.R. Olchanheski Junior, J. Padilha De Paula, S.F. Zawadzki, D. Fernandes, P.V. Farago, PCL/PHBV microparticles as innovative carriers for oral controlled release of manidipine dihydrochloride, Sci. World J. 268107 (2014) 1-10.

[22] C. Caleja, L. Barros, A.L. Antonio, M.B.P.P. Oliveira, I.C.F.R. Ferreira, A comparative study between natural and synthetic antioxidants: evaluation of their performance after incorporation into biscuits, Food Chem. 216 (2017) 342-346.

[23] C.F. Kuo, C.H. Hsieh, W.Y. Lin, Proteomic response of LAP-activated RAW 264.7 macrophages to the anti-inflammatory property of fungal ergosterol, Food Chem. 126 (2011) 207-212.

[24] W. Thangboonjit, S. Limsaeng-u-rai, U. Panich, Comparative evaluation of antityrosinase and antioxidant activities of dietary phenolics and their activities in melanoma cells exposed to UVA, Siriraj Med. J. 66 (2014) 5-10.

[25] S.M. An, J.S. Koh, Y.C. Boo, p-Coumaric acid not only inhibits human tyrosinase activity in vitro but also melanogenesis in cells exposed to UVB, Phyther. Res. 24 (2010) 1175-1180.

[26] H. jin Jun, J.H. Lee, B.R. Cho, W.D. Seo, D.W. Kim, K.J. Cho, S.J. Lee, p-Coumaric acid inhibition of CREB phosphorylation reduces cellular melanogenesis, Eur. Food Res. Technol. 235 (2012) 1207-1211.

[27] S.G. Parkar, D.E. Stevenson, M.A. Skinner, The potential influence of fruit polyphenols on colonic microflora and human gut health, Int. J. Food Microbiol. 124 (2008) 295-298.

[28] C. Cueva, M.V. Moreno-Arribas, P.J. Martín-Álvarez, G. Bills, M.F. Vicente, A. Basilio, C.L. Rivas, T. Requena, J.M. Rodríguez, B. Bartolomé, Antimicrobial activity of phenolic acids against commensal, probiotic and pathogenic bacteria, Res. Microbiol. 161 (2010) 372-382.

[29] T. Pluemsamran, T. Onkoksoong, U. Panich, Caffeic acid and ferulic acid inhibit UVA-induced matrix metalloproteinase- 1 through regulation of antioxidant defense system in keratinocyte HaCaT cells, Photochem. Photobiol. 88 (2012) 961-968.

[30] J.K. Seok, Y.C. Boo, P-coumaric acid attenuates UVB-induced release of stratifin from keratinocytes and indirectly regulates matrix metalloproteinase 1 release from fibroblasts, Korean J. Physiol. Pharmacol. 19 (2015) 241-247.

[31] J. Aguiar, R. Costa, F. Rocha, B.N. Estevinho, L. Santos, Design of microparticles containing natural antioxidants: preparation, characterization and controlled release studies, Powder Technol. 313 (2017) 287-292.

[32] T.S. Chang, An updated review of tyrosinase inhibitors, Int. J. Mol. Sci. 10 (2009) 2440-2475.

[33] Y.K. Seo, S.J. Kim, Y.C. Boo, J.H. Baek, S.H. Lee, J.S. Koh, Effects of p-coumaric acid on erythema and pigmentation of human skin exposed to ultraviolet radiation, Clin. Exp. Dermatol. 36 (2011) 260-266.

[34] J.V. Andhariya, S. Choi, Y. Wang, Y. Zou, D.J. Burgess, J. Shen, Accelerated in vitro release testing method for naltrexone loaded PLGA microspheres, Int. J. Pharm. 520 (2017) 79-85.

[35] T. Biswick, D.H. Park, J.H. Choy, Enhancing the UV A1 screening ability of caffeic acid by encapsulation in layered basic zinc hydroxide matrix, J. Phys. Chem. Solids 73 (2012) 1510-1513.

[36] M.A. Ouimet, J.J. Faig, W. Yu, K.E. Uhrich, Ferulic acid-based polymers with glycol functionality as a versatile platform for topical applications, Biomacromolecules. 16 (2015) 2911-2919.

[37] N. Sharma, P. Madan, S. Lin, Effect of process and formulation variables on the preparation of parenteral paclitaxel-loaded biodegradable polymeric nanoparticles: a co-surfactant study, Asian J. Pharm. Sci. 11 (2016) 404-416.

[38] J.S. and D.J. Burgess, Accelerated in vitro release testing methods for extended release parenteral dosage form, J Pharm Pharmacol. 64 (2012) 986-996.

[39] S.M. Ali, G. Yosipovitch, Skin pH: from basic science to basic skin care, Acta Derm. Venereol. 93 (2013) 261-267.

[40] J.J. Martínez-Pla, Y. Martín-Biosca, S. Sagrado, R.M. Villanueva-Camañas, M.J. Medina-Hernández, Evaluation of the $\mathrm{pH}$ effect of formulations on the skin permeability of drugs by biopartitioning micellar chromatography, J. Chromatogr. A 1047 (2004) 255-262. 